ULB-TH-98/18

\title{
Leptogenesis with virtual Majorana neutrinos
}

\author{
J.-M. Frère, F.-S. Lingt, M. H.G. Tytgat? and V. Van Elewyckę \\ Service de Physique Théorique, CP225 \\ Université Libre de Bruxelles \\ Bvd du Triomphe, 1050 Brussels, Belgium
}

\begin{abstract}
We present a mechanism of leptogenesis based on the out-of-equilibrium decay of a scalar particle into heavy virtual Majorana neutrinos. This scheme presents many conceptual advantages over the conventional scenario of Fukugita and Yanagida. In particular, the standard techniques of quantum field theory can be used to compute the lepton asymmetry, without resorting to the phenomenological approximations usually made to describe unstable particles. This simplification allows us to address in a well-defined framework some issues raised in the recent literature. We also show, in a toy model, that a successful leptogenesis scenario is possible and requires a rather light scalar particle, $10^{6} \mathrm{GeV}<m<10^{13} \mathrm{GeV}$. A natural embedding of this scheme in a gauged unified theory encompassing the Majorana fermions seems however difficult

\footnotetext{
${ }^{1}$ Aspirant FNRS.

${ }^{2}$ Collaborateur Scientifique FNRS.

${ }^{3}$ Chercheur FRIA.
} 


\section{Introduction}

Leptogenesis is an attractive scenario for the origin of the baryon number of the Universe. It rests on the idea that if an antilepton excess is created at a scale well above the electroweak phase transition, $T \gg 100 \mathrm{GeV}$, it can be very efficiently converted into a net baryon asymmetry by spaleron-like processes, that violate $B+L$ but preserve $B-L$. In the simplest scenarios, the initial lepton asymmetry is created in the out-of-equilibrium decay of heavy Majorana neutrinos [1]. Among other things, such a scheme has the advantage of separating the step of $C P$ and $L$ violation from the step of $B$ violation, which occurs later through sphaleron-like processes, and thus to avoid the pitfalls of maintaining an out-of-equilibrium situation around the electroweak scale. (See i.e. [2] for a review of electroweak baryogenesis scenarios.) Also, as the conversion of $L$ into $B$ takes place at equilibrium and is essentially complete, these mechanisms are largely insensitive to the details of the non-perturbative baryon number violating processes.

As is well known, CP violation is a crucial ingredient of leptogenesis [3] and it here arises from the interference between tree-level diagrams and the absorptive part of one-loop diagrams. Traditionally, only the one-loop vertex corrections were taken into account in most calculations [4], even though it was known [5] that the self-energy corrections, through which the different Majorana neutrinos can mix, do also contribute to the CP asymmetry. In particular, in the framework of the wave-function formalism of Weisskopf and Wigner [6], it has been argued that, in the limit of nearly degenerate Majorana neutrinos, the self-energy contribution could be significantly larger than

the vertex term, thus giving an enhanced lepton asymmetry [7, 8, 9]. Using the exact solution of the wave equation with a complex matrix, this effect has been verified for the case of two scalar flavours [10]. The wave-function approach is only a phenomenological approximation however, and one might 
wish for a more rigorous and systematic formulation. The problem, as is well-known, is that unstable particles are outside the realm of conventional quantum field theory, as they cannot be asymptotic states of the S-matrix. In particular, the self-energy corrections cannot be absorbed into the field wave-function renormalization constant, without destroying the hermiticity of the lagrangian.f Another, but related, issue is that the Majorana propagation eigenstates are not well-defined, an effect that leads to an ambiguity in the initial conditions for leptogenesis.尸

To address some of these problems, it has been proposed in 12 to consider lepton number violating scattering processes in which the Majorana particles appear only in intermediate states, like in

$$
l_{L} \phi \rightarrow N^{*} \rightarrow l_{L}^{c} \phi^{\dagger}
$$

where $l_{L}$ are the left-handed Standard Model (SM) leptons, $\phi$ is the Higgs doublet and $N$ are off-shell $\left(^{*}\right)$ Majorana neutrinos. Compared to the Majorana decay, the main advantage of considering processes like (1.1) is that the rules of quantum field theory can be applied straightforwardly. It is for instance manifest that the self-energy corrections to the propagator of the Majorana must be included at one-loop. What is less obvious is how much these corrections contribute to the lepton asymmetry. Actually, as has been shown in [13], unitarity implies that when all the scattering channels like (1.1) are taken into account, the resulting lepton excess is precisely zero. This is actually just the requirement of departure from equilibrium: as the initial and final states in the processes (1.1) are the same, at equilibrium no lepton asymmetry can be created. Departure from equilibrium can be provided by the expansion of the Universe, that effectively selects a subset of the processes (1.1) and can thus lead to a non-vanishing lepton asymmetry [13, 9].

\footnotetext{
${ }^{4} \mathrm{~A}$ more satisfying approach, that only slightly departs from the canonical rules of quantum field theory, has been advocated in [11].

${ }^{5}$ This has been emphasized in 10] for instance, but presumably is a well-known problem.
} 
In the present paper, we study a different mechanism, that is a variation on the scattering scenario of [12]. In section 2, we will consider a heavy scalar particle, $\chi$, that is allowed to decay into light (unspecified but sterile) righthanded fermions and heavy right-handed Majorana neutrinos. The Majorana neutrinos decay into the left-handed SM leptons and Higgs scalar. (See figure 1.) If we impose the mass hierarchy,

$$
m_{M} \gg m_{\chi} \gg m_{\phi}, m_{l}=0
$$

the $\chi$ can be viewed as a source of Majorana neutrinos. This scenario encompasses the conceptual advantages of the scattering processes (1.1) but furthemore leads to the production of a net lepton asymmetry. Among other things, we will verify that the self-energy corrections do indeed give a nonnegligible contribution to the asymmetry. We will study the limit of degenerate Majorana neutrino masses, and show that the asymmetry has a finite, well-defined expression at one-loop. (Phase counting shows that even in this case, CP violation effects are possible.) Also, the asymmetry so obtained is directly related to the initial abundance of the $\chi$, independently of the basis chosen to define the Majorana states. We provide some numerical calculation performed for two flavours that make the various contributions (vertex and self-energy) to the asymmetry more explicit and give some useful estimates. Finally, we discuss the out-of-equilibrium conditions in the Early Universe, that puts limits on the Majorana and $\chi$ masses. These constraints compel the leptogenesis scenario to involve a neutral scalar $\chi$ at a scale between $10^{6}$ and $10^{13} \mathrm{GeV}$.

In Section 3, we try to embed our scheme in a more physically motivated framework. For definiteness, we have in mind a natural gauge extension of the model, for instance $S O(10)$. For simplicity, we have confined our argument to its subgroup $S U(2)_{L} \times S U(2)_{R} \times U(1)$, which already imposes strong constraints. As we will show, adding further gauge degrees of freedom 
has non-trivial consequences. In this framework, the $\chi^{+}$is the singly charged component of a triplet of $S U(2)_{R}$ while the vacuum expectation value of the neutral component $\chi^{0}$ breaks $S U(2)_{R}$ and gives a Majorana mass to the right-handed neutrinos,

$$
\Delta_{R} \equiv\left(\begin{array}{cc}
\chi^{+} / \sqrt{2} & \chi^{++} \\
\chi^{0} & -\chi^{+} / \sqrt{2}
\end{array}\right) .
$$

The decay of the $\chi^{+}$as the source of the lepton asymmetry is however immediately ruled out: the annihilation of $\chi^{+} \chi^{-}$pairs into photons is much too fast, so that the charged $\chi$ stay in thermal equilibrium at the epoch of interest, $T \sim m_{\chi}$. The next possibility is to consider the decay of the neutral $\chi^{0}$ (figure 8). Because the lepton number violating decay rate is relatively slow in this case, we have to consider other competing rare decay processes. As almost no dilution is allowed, an analysis of the dominant decay channels reveals that coupling of scalar particle to the $S U(2)_{R}$ gauge bosons is sufficient to totally damp out the lepton asymmetry. Adding more fields could resolve this problem, but at the price of simplicity.

\section{$2 \chi$ decay leptogenesis: self-energy and ver- tex corrections}

We first concentrate on the various sources of $\mathrm{CP}$ violation in the decay of the scalar particle $\chi$. The final state considered is a right-handed (sterile) fermion accompanied by a left-handed lepton and a Higgs boson, and reached through the exchange of a virtual heavy Majorana particle $\chi \rightarrow l_{R} N^{*} \rightarrow l_{L} l_{R} \phi$ (see diagram 1, figure 1). The Majorana neutrinos are labelled according to their mass; the following mass hierarchy guarantees that the intermediate Majorana neutrino is off mass-shell,

$$
M_{3}>M_{2}>M_{1}>m_{\chi} \gg m_{l}, m_{\phi}=0
$$


We want to study the particular consequences of this mass hierarchy on $\mathrm{CP}$ violation, as a theoretical framework and as a possible realistic scenario for leptogenesis.

The decay of the $\chi$ is expected to produce a lepton asymmetry, since the intermediate Majorana can couple to both lepton-antiboson and antileptonboson, with final total lepton number 2, 0 or -2 (figure 1). P The most general Yukawa lagrangian for the particles involved in our scheme is of the form

$$
\mathcal{L}_{y u k}=g_{i j} \bar{L}_{L i} \Phi R N_{j}+G_{k l} \chi \bar{l}^{c} R k R N_{l}+\text { h.c. }
$$

where $L_{L}$ stands for the left-handed SM leptons, $\Phi$ is the Higgs doublet, $N$ are the heavy Majorana neutrinos, $l_{R}$ are the light, sterile right-handed fermions, and $R=\frac{1+\gamma_{5}}{2}$. Conventionnally, the Majorana states $N$ are chosen so that the Majorana mass matrix $M$ is real and diagonal, which is always possible. The two Yukawa coupling matrices $g$ and $G$, however, cannot be diagonalised simultaneously with $M$, in general. The lagrangian (2.1) leads to the tree level decays for the $\chi$ of figure 1 . The decay channels form hermitian conjugate pairs, e.g. II and III, and CP violation becomes possible only at the one loop level, where tree level and one-loop diagrams can interfere. We expect that both loops including $\chi$ and $\phi$ scalars will contribute to the global asymmetry, since $\mathrm{CP}$ violating phases appear in the coupling matrices $g$ and $G$ in the most general case. We can split the one-loop diagrams into self-energy loops and vertex corrections. While a calculation including the vertex correction alone is well known to yield a non-vanishing asymmetry, the self-energy correction has been often neglected. However, it has been argued in [8] and [9] that its contribution is far from negligible, and is even enhanced

\footnotetext{
${ }^{6}$ This assignment corresponds to total lepton number, left-handed plus right-handed. If the right-handed fermions are sterile, only the left-handed lepton number matters for leptogenesis. Our conclusions are essentially independent of the charge assignement chosen.
} 


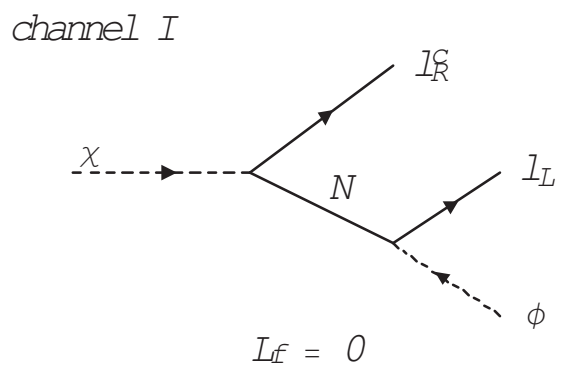

channel II

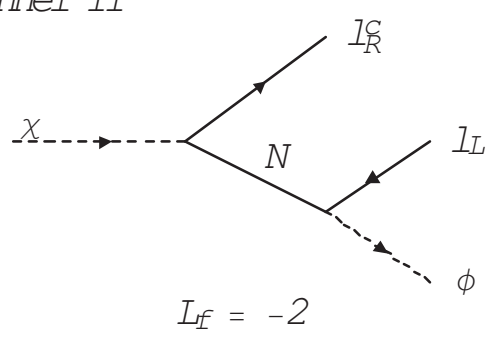

channel IV

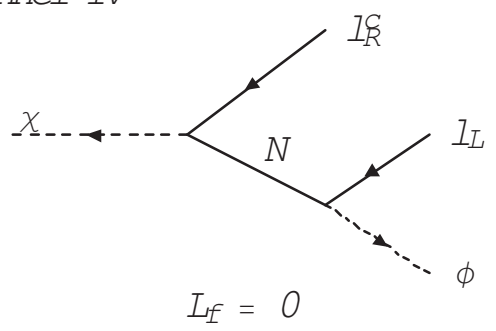

channel III

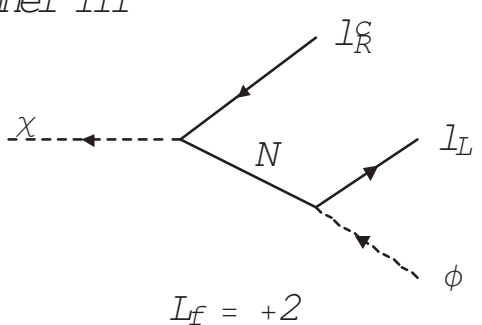

Figure 1: Decay channels of $\chi$

for nearly degenerate Majorana masses. In our scheme, the inclusion of selfenergy loops is automatic since the Majorana neutrinos only appear as virtual intermediate states.

According to the Cutkosky rules, the absorptive part of the one-loop diagrams, which provides the imaginary part needed for the CP asymmetry, are given by the cut diagrams. Diagrams $1,2,5$ of figure 2 are purely dispersive (no unitarity cut operates) and therefore don't contribute to the $\mathrm{CP}$ asymmetry. In particular, the self-energy correction of diagram 5 can be absorbed in the wave-function renormalisation of the $\chi$. Consequently, in this scheme, the sources of $\mathrm{CP}$ violation are precisely the same as those relevant in the conventional Majorana neutrinos decay scenario, namely, the asymmetry comes from loops involving the Higgs scalar.

At tree level, the $\chi$ decay rate is

$$
\Gamma_{0}\left[\chi \rightarrow l_{R j} l_{L k} \bar{\phi}\right]=\frac{m_{\chi}}{64(2 \pi)^{3}} \sum_{i, l}\left(g^{\dagger} g\right)_{l i}\left(G^{\dagger} G\right)_{l i} f^{(0)}\left(\frac{M_{i}}{m_{\chi}}, \frac{M_{l}}{m_{\chi}}\right)
$$



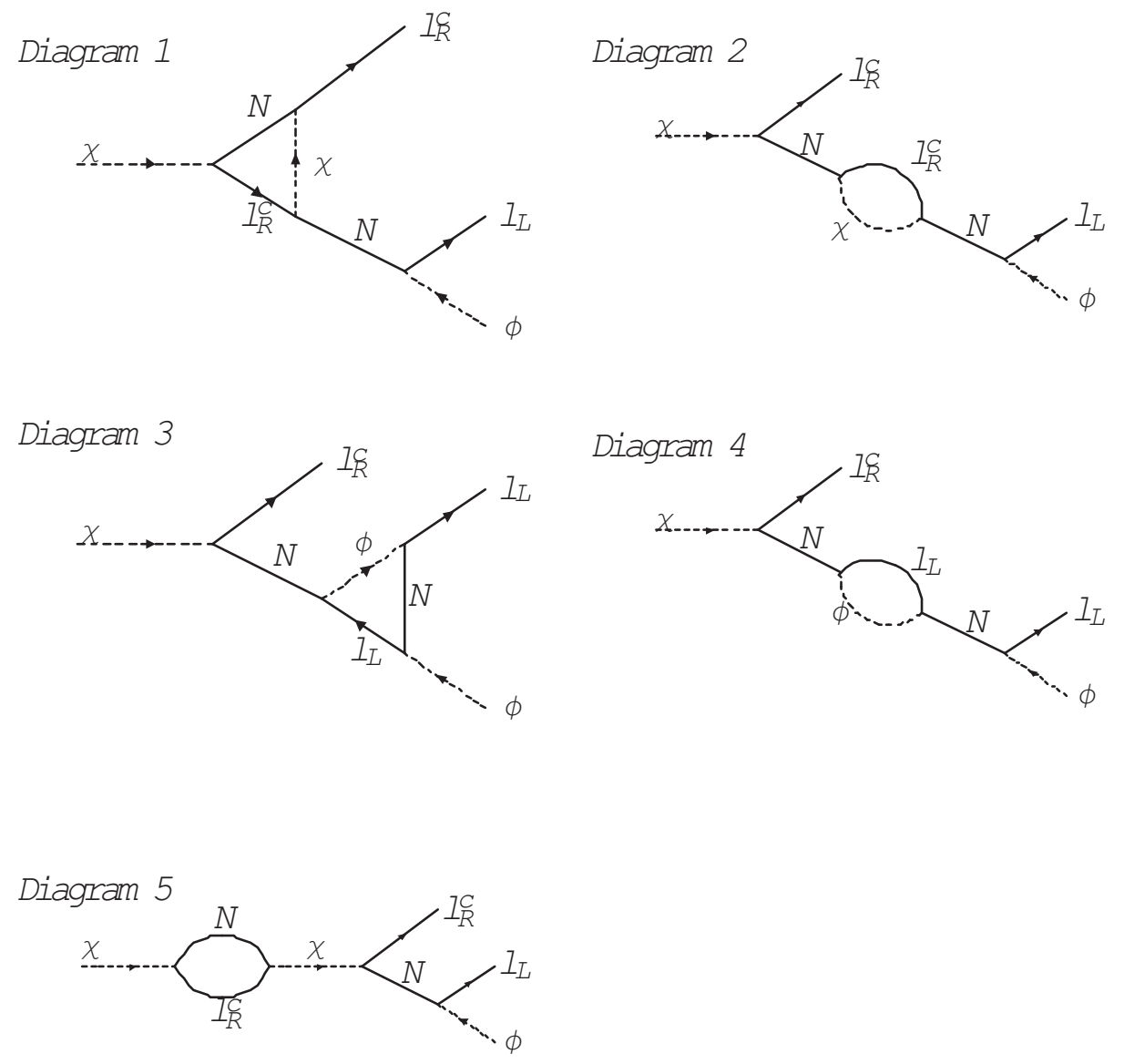

Figure 2: One-loop corrections.

where

$$
f^{(0)}(x, y)=x y\left(1+\frac{\left(x^{2}-1\right)^{2}}{x^{2}-y^{2}} \ln \left(1-\frac{1}{x^{2}}\right)-\frac{\left(y^{2}-1\right)^{2}}{x^{2}-y^{2}} \ln \left(1-\frac{1}{y^{2}}\right)\right)
$$

This function has a well-defined limit when the Majorana are degenerate,

$$
\lim _{y \rightarrow x} f^{(0)}(x, y)=2 x^{2}-1+2 x^{2}\left(x^{2}-1\right) \ln \left(1-\frac{1}{x^{2}}\right) .
$$

while in the limit of very heavy Majorana neutrino $m_{\chi} \ll M$,

$$
\Gamma_{0}\left[\chi \rightarrow l_{R j} l_{L k} \bar{\phi}\right] \rightarrow \frac{m_{\chi}^{3}}{3 \cdot 2^{6}(2 \pi)^{3}} \sum_{i, l} \frac{\left(g^{\dagger} g\right)_{l i}\left(G^{\dagger} G\right)_{l i}}{M_{i} M_{l}}
$$


At this order, the CP asymmetry splits into two parts that can be calculated separately. The vertex correction gives the following contribution to the asymmetry,

$$
\epsilon_{v}=\frac{\Gamma_{01, v}^{a s .}\left[\chi \rightarrow l_{R j} l_{L k} \bar{\phi}\right]}{\Gamma_{0}\left[\chi \rightarrow l_{R j} l_{L k} \bar{\phi}\right]}
$$

with

$$
\Gamma_{01, v}^{a s .}\left[\chi \rightarrow l_{R j} l_{L k} \bar{\phi}\right]=\frac{m_{\chi}}{32(2 \pi)^{4}} \sum_{i, l, n} \Im m\left(g^{\dagger} g\right)_{n l}\left(g^{\dagger} g\right)_{n i}\left(G^{\dagger} G\right)_{i l} f_{v}\left(\frac{M_{i}}{m_{\chi}}, \frac{M_{l}}{m_{\chi}}, \frac{M_{n}}{m_{\chi}}\right)
$$

The function $f_{v}(x, y, z)$ is again well-defined for degenerate Majorana masses. In the limit $m_{\chi} \ll M$ as in Eq. (2.3) above,

$$
\Gamma_{01, v}^{a s .}\left[\chi \rightarrow l_{R j} l_{L k} \bar{\phi}\right] \rightarrow \frac{m_{\chi}^{5}}{3 \cdot 2^{10}(2 \pi)^{4}} \sum_{i, l, n} \frac{\Im m\left(\left(g^{\dagger} g\right)_{n l}\left(g^{\dagger} g\right)_{n i}\left(G^{\dagger} G\right)_{i l}\right)}{M_{i}^{2} M_{l} M_{n}}
$$

The self-energy correction gives

$$
\epsilon_{w}=\frac{\Gamma_{01, w}^{a s}\left[\chi \rightarrow l_{R j} l_{L k} \bar{\phi}\right]}{\Gamma_{0}\left[\chi \rightarrow l_{R j} l_{L k} \bar{\phi}\right]}
$$

with

$$
\Gamma_{01, w}^{a s .}\left[\chi \rightarrow l_{R j} l_{L k} \bar{\phi}\right]=\frac{m_{\chi}}{16(2 \pi)^{4}} \sum_{i, l, n} \Im m\left(g^{\dagger} g\right)_{n l}\left(g^{\dagger} g\right)_{n i}\left(G^{\dagger} G\right)_{i l} f_{w}\left(\frac{M_{i}}{m_{\chi}}, \frac{M_{l}}{m_{\chi}}, \frac{M_{n}}{m_{\chi}}\right)
$$

The complete expression for $f_{w}$ is cumbersome, but it can be expressed in terms of simple functions. Again, the limit of degenerate Majorana masses gives a well defined value,

$$
\lim _{M_{i}, M_{l}, M_{n} \rightarrow M} f_{w}\left(\frac{M_{i}}{m_{\chi}}, \frac{M_{l}}{m_{\chi}}, \frac{M_{n}}{m_{\chi}}\right)=\frac{1}{16}-\frac{3 M^{2}}{8 m_{\chi}^{2}}+\left(\frac{M^{2}}{4 m_{\chi}^{2}}+\frac{3 M^{4}}{8 m_{\chi}^{4}}\right) \ln \frac{M^{2}-m_{\chi}^{2}}{M^{2}}
$$

Also, in the limit of a light $\chi, m_{\chi} \ll M$,

$$
\Gamma_{01, w}^{a s .}\left(\chi \rightarrow l_{R j} l_{L k} \bar{\phi}\right) \rightarrow \frac{m_{\chi}^{5}}{3 \cdot 2^{9}(2 \pi)^{4}} \sum_{i, l, n} \frac{\Im m\left(\left(g^{\dagger} g\right)_{n i}\left(g^{\dagger} g\right)_{n l}\left(G^{\dagger} G\right)_{i l}\right)}{M_{n}^{2} M_{l} M_{i}}
$$


In the conventional scenario, with Majorana neutrinos in the initial state, there are unphysical singularities $\propto 1 /\left(M_{i}^{2}-M_{j}^{2}\right)$ in the expression of the self-energy term (see for instance [10] or [12]). They signal a breakdown of the perturbative expansion for $\Delta M \sim \Gamma$, where $M$ and $\Gamma$ are respectively the mass and width of the Majorana neutrinos, and are responsible for the enhancement of the lepton asymmetry from the self-energy contribution with respect to one from the vertex. The present scheme offers no such singularities, and the limit of degenerate Majorana neutrinos is finite, Eq. (2.9). This holds of course as long as the Majorana are off mass shell, which is guaranteed by our choice of mass hierarchy, because the mass of the $\chi$ particle limits the value of $q^{2}$ flowing through the Majorana propagator to be at most $m_{\chi}^{2} \ll M_{i}^{2}$. In the case of 2-body scattering of light particles, the self-energy correction presents the same pole, because the centre of mass energy in a scattering process is not limited in principle, even if this scattering is supposed to occur in the thermal bath at the temperature $T^{2} \ll M_{i}^{2}$, i.e. at the epoch when the lightest heavy Majorana neutrino decays. This pole enhances the contribution of the self-energy compared to the one from the vertex in the degenerate limit, but necessitates one to use either a quite elaborate resummation scheme (for instance as in [12] which is limited to weak mixing) or a wave-functional approach [10].

The absence of such singularities is another non-negligeable simplification offered by the present approach. On the other hand, there is no large enhancement $\sim M / \Gamma$ either (provided $\left.m_{\chi}<M\right)$. However, the asymmetry is well defined for all Majorana mass patterns, including the limit of degenerate Majorana masses, for which the asymmetry does not necessarily vanish.

Let us also re-emphasise that, contrarily to the scattering processes of (1.1) studied in [12, 13], a true lepton asymmetry can be produced here. This is simply because the $\chi$ is unstable below $T<m_{\chi}$ while the inverse decay, or recombinations is Boltzmann suppressed, which is another way to state that 
the unitarity constraint does not apply. Turning now to leptogenesis, the framework of this mechanism is just the basic out-of-equilibrium decay of unstable particles. The various rates and CP asymmetries can be computed without reference to the Majorana propagation eigenstates. We only have to ensure that the conditions of an out-of-equilibrium decay for the $\chi$ are verified.

We have performed an explicit (numerical) calculation of the ratio between the self-energy and the vertex contributions to the asymmetry, in the special case of two flavours. In the restricted parameter area spanned by the two lightest Majorana neutrinos, the matrices appearing in the expressions of the asymmetries can be parameterised as follows: the Majorana mass matrix can always be set to a real positive diagonal form

$$
M=\left(\begin{array}{cc}
M_{1} & 0 \\
0 & M_{2}
\end{array}\right)
$$

while $g$ is a complex matrix. As the product $g g^{\dagger}$ can be diagonalized by a unitary transformation on the left-handed leptons, we can parameterise $g$ as

$$
g=\left(\begin{array}{cc}
g_{1} e^{i \alpha} & -\frac{g_{0}^{2}}{g_{2}} e^{-i \beta} \\
\frac{g_{0}^{2}}{g_{1}} e^{i \beta} & g_{2} e^{-i \alpha}
\end{array}\right),
$$

There is a similar parameterisation for $G$. Already with two flavours, there are four $\mathrm{CP}$ violating phases, even in the degenerate case, because the two Yukawa coupling matrices limit the redefinition freedom on the right-handed neutrinos. This has to be contrasted with the conventional scenario, where only the two matrices $M$ and $g$ are present, so that an appropriate unitary rotation $U_{R}$ can eliminate the two $\mathrm{CP}$ violating phases of $g$ in the degenerate case. In the present case, these two phases would only be moved from $g$ to $G$. Of course, if $G$ is proportional to $g, M$ or the identity matrix, both the vertex and self-energy contributions to the asymmetry vanish. (As shown in [15, even with the matrices $M$ and $g$ alone, one $\mathrm{CP}$ violation phase 
can remain in the mass-degenerate limit if all three flavours are taken into account.) Figure 3 presents the ratio $r=\epsilon_{w} / \epsilon_{v}$ plotted against the mass hierarchy $h=M_{2} / M_{1}$, in the case where the $\chi$ particle mostly couples to the lighter Majorana neutrino. For large mass splitting, i.e. when $h \gg 1$,

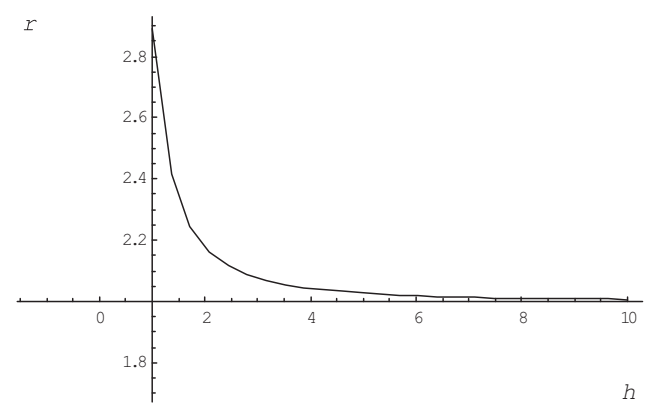

Figure 3: Ratio $r=\epsilon_{w} / \epsilon_{v}$ vs mass ratio $h=M_{1} / M_{2}$.

the self-energy term becomes twice the vertex one, $r \rightarrow 2$. This limit was previously obtained in [10] for scalar neutrinos decays. It is always true in the case $m_{\chi} \ll M$, as can be verified in the equations (2.6) and (2.10). But in the general case, the asymptotic value of this ratio depends on the coupling matrices elements. On the other hand, in the degenerate case, the self-energy contribution doesn't generally vanish, and can even have its maximum value at this point. The shape of the ratio $r$ against $h$ can be very different according to the choice of the coupling matrix $G$, but generally, the self-energy contribution to the CP asymmetry is non-vanishing, even in the mass-degenerate limit. Also, as already stated, to consider the Majorana neutrinos as intermediate states only permits to circumvent the problem of defining the propagation eigenstates. The current attempts to define the external states (see [10] and [12 for example) are based on some diagonalisation which necessarily uses non-unitary transformations so that the resulting propagation eigenstates don't have a physical significance.

Before going on, it might be of interest to see why the cancellations met 
in the 2-body scattering scenario of [12] do not occur here. This can be seen from the diagrammatic representation of "cut blobs", following [13]. To be concrete, we have chosen

$$
G=\left(\begin{array}{ll}
1 & 0 \\
0 & 0
\end{array}\right)
$$

i.e. $\chi$ is only coupled to $N_{1}$. In figures 4 and 5 , we have only drawn the

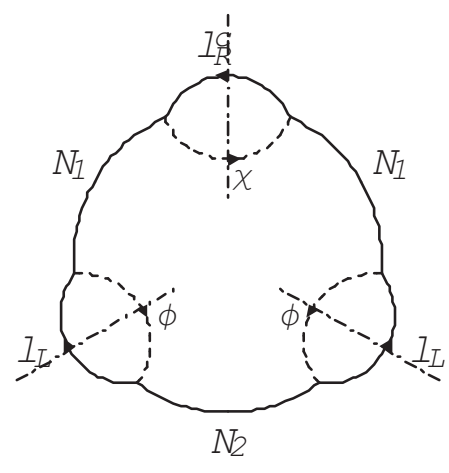

$I_{f}=-2$

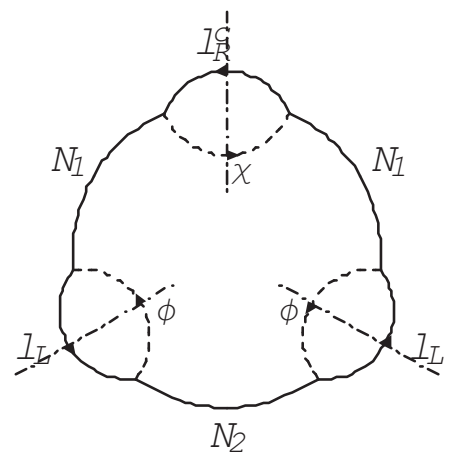

$L_{f}=-2$

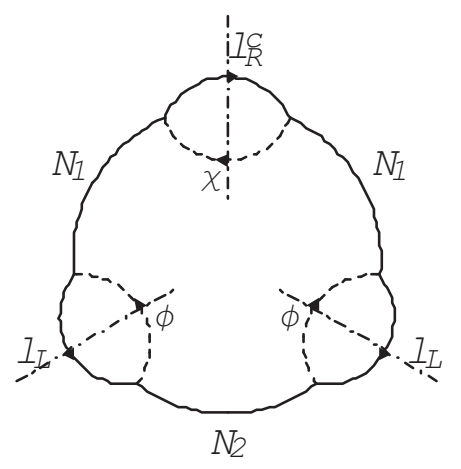

$I_{f}=+2$

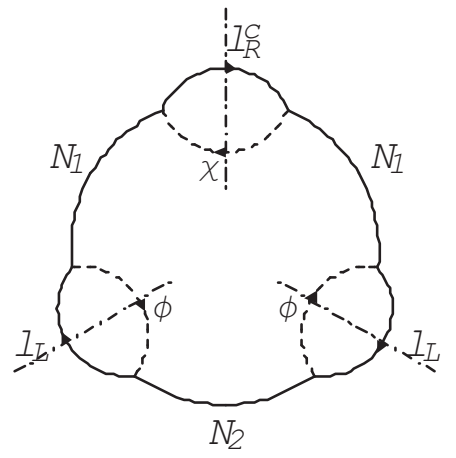

$L_{f}=+2$

Figure 4: Interference diagrams with $\left|L_{f}\right|=2$

diagrams for which the cancellation with conjugates] is not immediate. The

${ }^{7}$ The blobs of the second columns are the conjugate of those of the first columns. Note that the left-handed lepton current in the self-energy correction flips sign between the first and second row. 


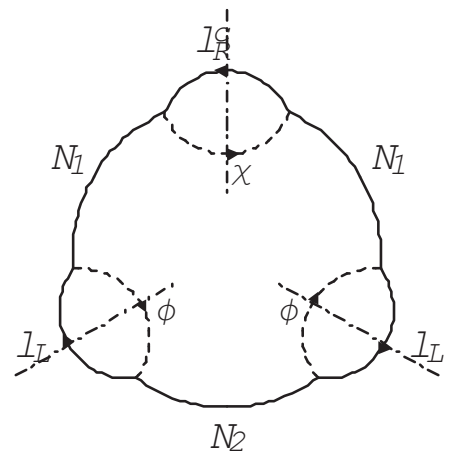

$I_{f}=0$

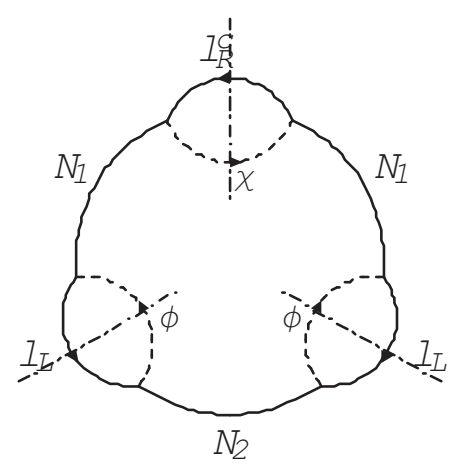

$L_{f}=0$

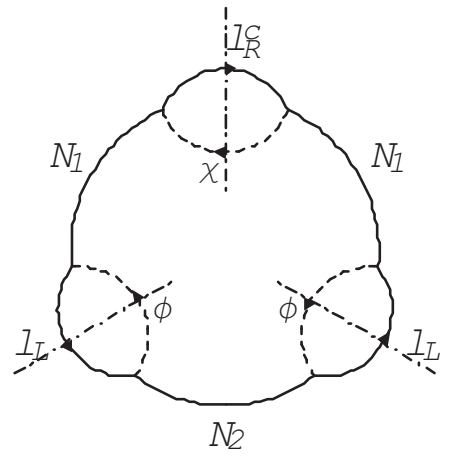

$L_{f}=0$

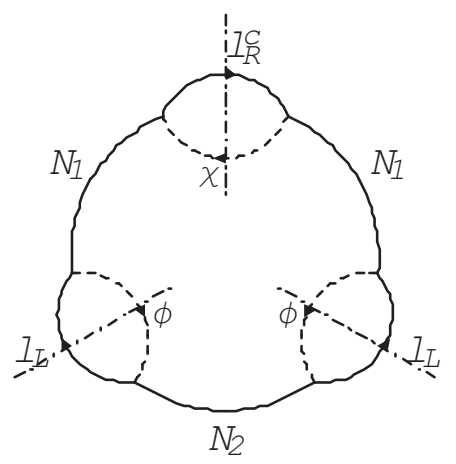

$I_{f}=0$

Figure 5: Interference diagrams with $L_{f}=0$

one-loop self-energy diagram consists of two parts, one with the left-handed lepton and Higgs scalar running one way, and the other with the arrows reversed. Then, the diagram with the lepton in the self-energy loop running from $N_{2}$ to $N_{1}$ cancels with its CP conjugate, while the other diagram must be added to the one with $L_{f}=0$ to find a complete cancellation. Hence, only when all the decay channels of the $\chi$ particle are taken into account, is there a full cancellation of the various (unweighted) CP self-energy asymmetries, but the lepton asymmetry produced by the self-energy in the decay channel does not vanish. This reasoning shows that, as expected, the lepton asymmetry 
is produced through the interference between $L=0$ and $L \neq 0$ channels, and that $\mathrm{CP}$ violation is observed only when specific channels (namely those with $L \neq 0$ are selected. Finally, like in the scattering processes (1.1), a full cancellation of the lepton asymmetry occurs if the inverse decay channels are taken into account 13. In the early universe, these channels are Boltzmann suppressed if the $\chi$ decays out-of-equilibrium.

The last question is to see whether our scheme can yield enough lepton asymmetry in the early universe. At this point, we must impose that the $\chi$ is a neutral particle, and for simplicity, we will assume a single real scalar, that couples to heavy Majorana neutrinos and some light, sterile right-handed fermions. Indeed, if the $\chi$ particles were charged, they would pair-annihilate very efficently into photons at $T \sim m_{\chi}$. The resulting lepton asymmetry would be Boltzman suppressed and too small for practical purposes.

From our calculations, a lepton excess $n_{L}=L / s \sim 10^{-10}$, where $s$ is the entropy density of the universe at the epoch of interest, can be reached for $x=m_{\chi} / M>10^{-2}$. Figure 6 shows for instance a plot of the two contributions, vertex and self-energy, to the asymmetry in the case of degenerate Majorana mass, which gives the largest asymmetry for a fixed scalar mass. As the value of the asymmetry increases when the scalar mass approaches
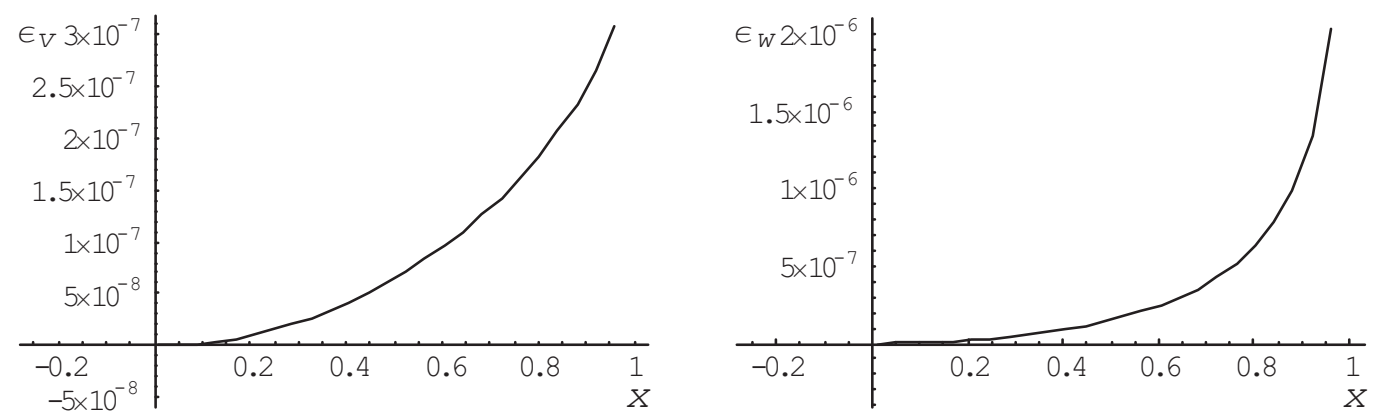

Figure 6: Asymmetry $\left|\epsilon_{v}\right|$ and $\left|\epsilon_{w}\right|$ versus $x=m_{\chi} / M$

the Majorana mass, it seems that it would suffice to take these as close 
as possible to maximise the lepton asymmetry. For leptogenesis, this has however a limitation. Suppose that the temperature is about the mass of the lightest Majorana neutrino $N_{1}$ and that $N_{1}$ is out-of-equilibrium. Then, since the 2-body lepton number violating scattering $l_{L} \bar{\phi} \leftrightarrow \overline{l_{L}} \phi$ is also outof-equilibrium, the decay of the Majorana neutrinos would also produce a net lepton asymmetry. In the thermal bath, this process will compete with the scalar decay. In that case, an estimate for the asymmetry must take the coupled evolution of both Majorana neutrinos and scalars into account.

For definiteness, we have chosen to consider a mechanism of leptogenesis where $L$ is evaluated from $\chi$ decays alone. We thus impose the following conditions,

$$
\begin{aligned}
& \alpha_{g} M>g_{*}^{1 / 2} \frac{M^{2}}{M_{\text {Planck }}} \\
& \alpha_{g}^{2} \frac{m_{\chi}^{3}}{M^{2}}<g_{*}^{1 / 2} \frac{m_{\chi}^{2}}{M_{\text {Planck }}} \\
& \alpha_{g} \alpha_{G} \frac{m_{\chi}^{3}}{M^{2}}<g_{*}^{1 / 2} \frac{m_{\chi}^{2}}{M_{\text {Planck }}} \\
& r=\frac{m_{\chi}}{M}>10^{-2}
\end{aligned}
$$

Equation (2.11) guarantees that the lightest Majorana $N_{1}$ is in thermal equilibrium when $T \sim M_{1}$, so that its decay does not generate any lepton asymmetry. Equation (2.12) ensures that all 2-body lepton number violating processes are out-of-equilibrium when the $\chi$ start to decay. Equation (2.13) is the requirement that $\chi$ decays out-of-equilibrium. Lastly, (2.14) is needed to have enough lepton asymmetry, .

These four constraints limit the mass of the Majorana and $\chi$ to be within the domain $D$ depicted in figure 7. (We have also imposed $m_{\chi}<M_{M}$ and we have taken $\alpha_{g} \equiv g^{2} / 4 \pi$ and $\alpha_{G} \equiv G^{2} / 4 \pi \sim 10^{-4}$ while $g_{*} \sim 10^{2}$ counts the number of degrees of freedom at the epoch of interest.) The allowed domain is quite large and not very constraining for the values of $m_{\chi}$. The constraint 


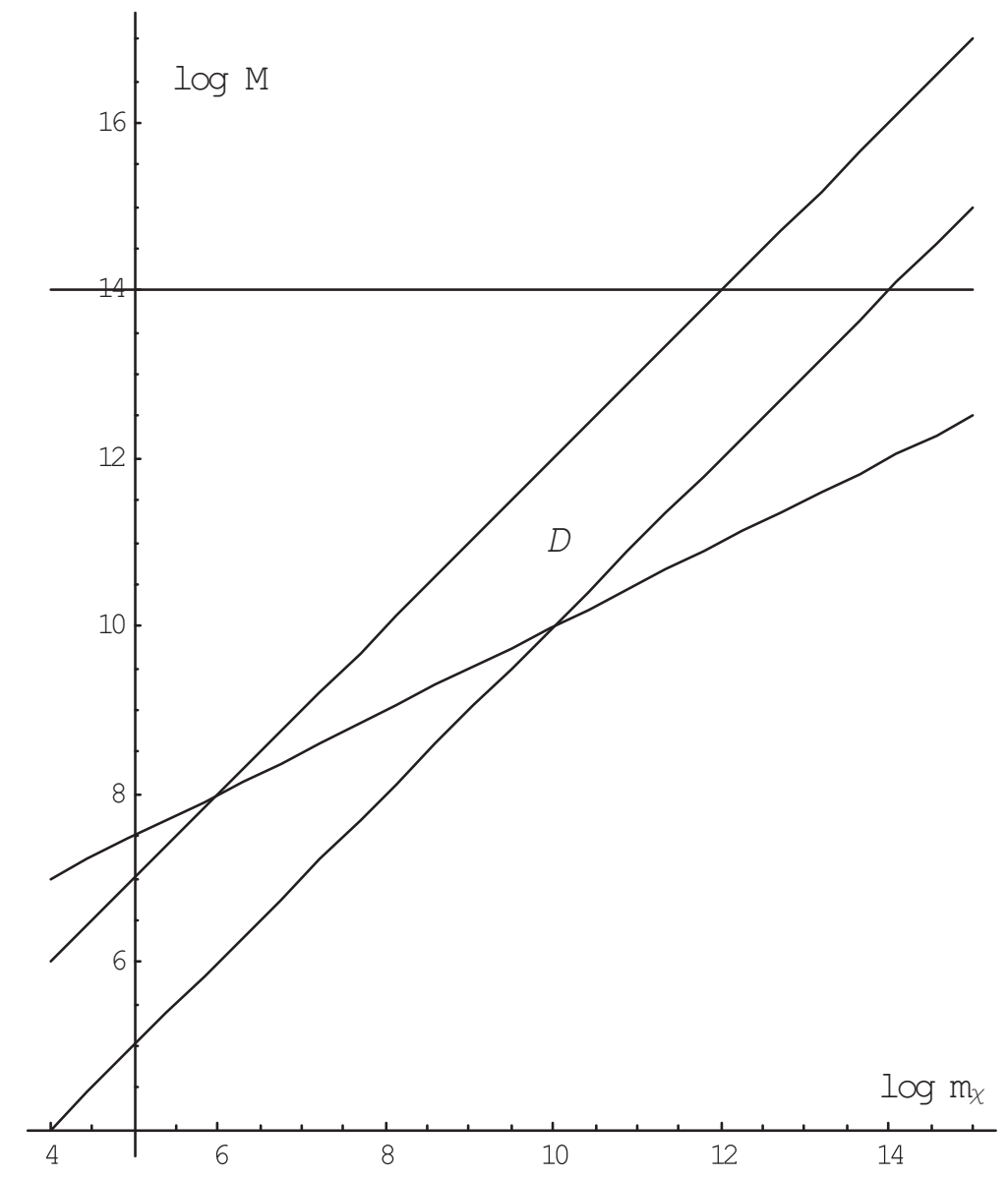

Figure 7: Mass scales constraints in log-log plot

from $n_{L} \sim 10^{-10}$ gives a lower bound on the scalar mass, $m_{\chi}>\sim 10^{6} \mathrm{GeV}$. In the conventional Majorana decay scenario there is a lower bound on the Majorana mass imposed by the condition that the neutrino decays out-ofequilibrium; in contrast, because we consider a three-body decay process which has a different energy dependence, the same condition gives here an upper bound on the mass of the $\chi, m_{\chi} \ll 10^{13} \mathrm{GeV}$.

From the discussion of the present section, it appears that our toy model provides a consistent mechanism of leptogenesis. The existence of a scalar 
coupled to Majorana neutrinos is however suggestive of an extension of the SM to $S O(10)$ or at least its subgroup $G=S U(2)_{L} \times S U(2)_{R} \times U(1)$ for instance. We analyze in section 3 how our model would fare in such a scheme.

\section{Effects of dilution in the gauged version of the model}

The simplest way to embed the $\chi$ particle into the framework of a gauge theory is to work in a left-right model, i.e. with the group $S U(2)_{L} \times S U(2)_{R} \times$ $U(1)$. The scalar sector contains (at least) one complex scalar $\Delta_{R} \sim(0,1,2)$ which is in a (complex) triplet representation of $S U(2)_{R}$, similar scalars that couple to $S U(2)_{L}, \Delta_{L} \sim(1,0,2)$ and a Higgs bi-doublet $\Phi \sim(1 / 2,1 / 2,0)$.

$$
\Delta_{R} \equiv\left(\begin{array}{cc}
\chi^{+} / \sqrt{2} & \chi^{++} \\
\chi^{0} & -\chi^{+} / \sqrt{2}
\end{array}\right)
$$

The Yukawa coupling of $\Delta_{R}$ to the right-handed lepton doublet $L_{R}$ is given by

$$
\mathcal{L}=-G \overline{L_{R}^{c}} \Delta_{R} L_{R}+\text { h.c. }
$$

The charged component $\chi^{+}$and the neutral component $\chi^{0}$ couple to the right-handed neutrinos while the doubly charged $\chi^{++}$only couples to charged leptons. In this scheme, the vacuum expectation value $\left\langle\chi^{0}\right\rangle=V_{R}$ of the neutral component gives the Majorana mass term to the right-handed neutrinos (we will take it to br real). As it is possible to choose a basis in which the Majorana mass matrix is real diagonal, the coupling of the right-handed lepton doublet to the scalar triplet is then also real diagonal (in the case of one triplet).

$$
G=\frac{1}{V_{R}}\left(\begin{array}{ccc}
M_{1} & 0 & 0 \\
0 & M_{2} & 0 \\
0 & 0 & M_{3}
\end{array}\right)
$$


In the broken phase, the remaining gauge group is $S U(2)_{L} \times U(1)$. The real, massive $\chi^{0}$ couples to two Majorana neutrinos, and the $\chi^{+}\left(\right.$the $\left.\Im m \chi^{0}\right)$ component is the longitudinal component of the massive gauge boson $W_{R}$ $\left(Z_{R}\right)$. As the $W_{R}$ (or the Goldstone $\chi^{+}$) are charged particles, they can annihilate into photons, and this process occurs much faster than the decay into light leptons, so that, as already stated in section 2, these particles are not suitable for leptogenesis. $Z_{R}$, being a heavy gauge boson, also abondantly decays into fermion pairs, and its decay leads to inacceptable dilution of the $\mathrm{CP}$ asymmetry in this channel.

If we want leptogenesis to occur in this minimal framework, we have to turn to the decay modes of the $\chi^{0}$, that occurs through two intermediate Majorana neutrinos, and yields four light particles in the final state (see figure 8).

$$
\mathcal{L}_{\chi}=G \bar{N} \chi N
$$

The problem we have to face now is that a four-body decay rate is generally quite small. We estimate that

$$
\Gamma_{0}(\chi \rightarrow l l \overline{\phi \phi}) \sim 10^{-7} \sum_{l, j}\left(g^{\dagger} g\right)_{l j}^{2} \frac{m_{\chi}^{5}}{M_{j} M_{l} V_{R}^{2}}
$$

The lepton asymmetry produced in this case is of the same order as the one obtained for the three-body decay discussed in section 2. Consequently, only a small dilution of the lepton asymmetry by the other, lepton-number conserving, decay channels of the $\chi^{0}$ is acceptable.

For one thing, this requires some fine tuning in the Higgs sector to prevent the $\chi^{0}$ to decay into other light scalars. But much more problematic are the other rare decay channels of the $\chi^{0}$ that can occur at one-loop, or through higher dimensional operators. leptons (figure 10). Consider for instance the one-loop decay mode of the $\chi^{0}$ into two photons (or two gauge bosons $a^{\mu}$ associated with $\left.U(1)_{Y}\right)$ as in figure 9 . This case is similar to the decay of the 

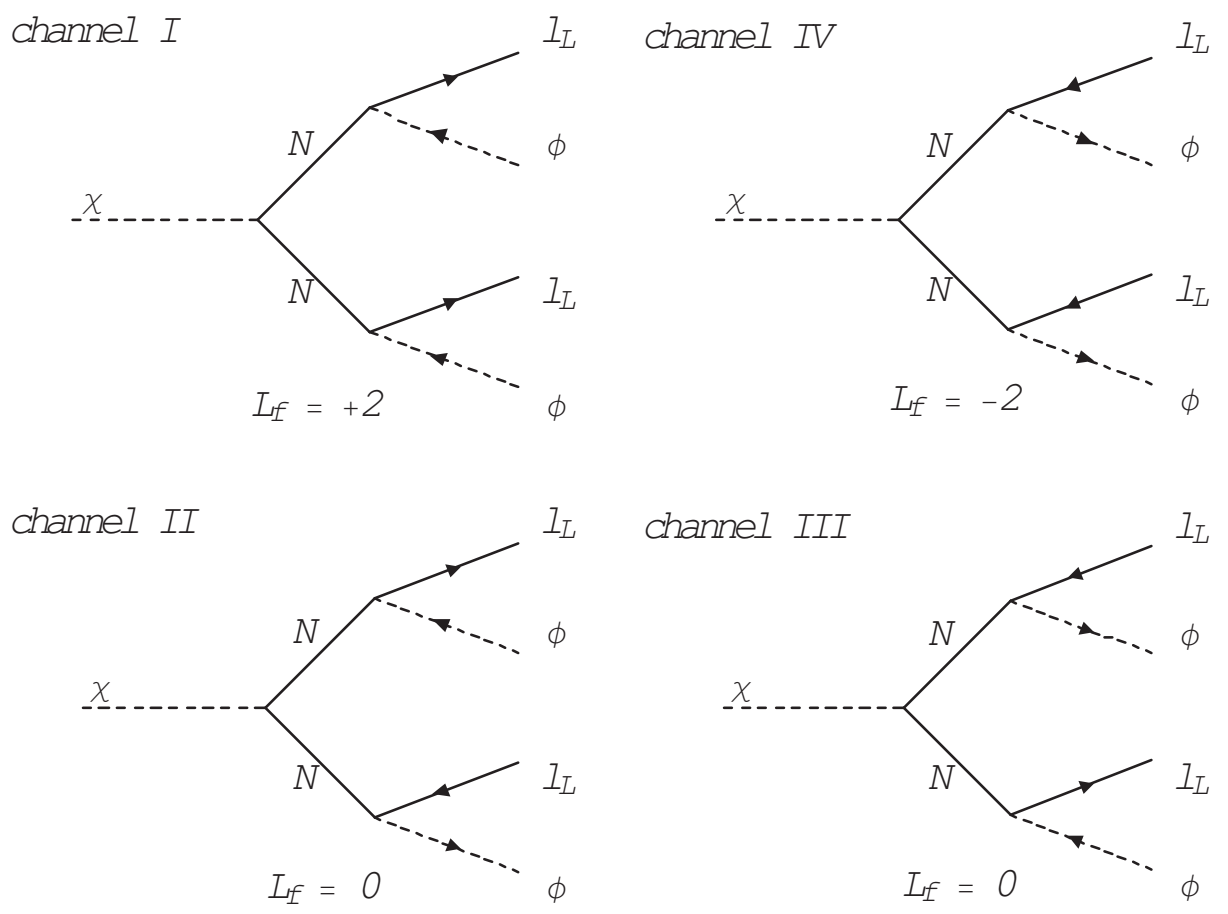

Figure 8: 4-body decays of the neutral $\chi$ in the $L R$ symmetric model
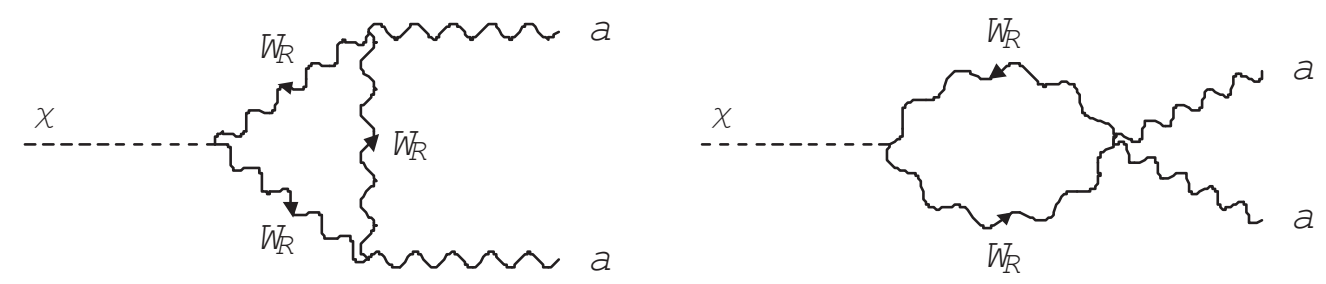

Figure 9: decay of $\chi$ to massless gauge bosons at one-loop level

Higgs into photons in the SM, which has been computed in [16]; it would give

$$
\Gamma_{\chi \rightarrow \gamma \gamma} \sim 10^{-5} g_{R}^{6} \frac{m_{\chi}^{3}}{M_{W}^{2}}
$$

$g_{R}$ stands for the coupling constant of the right-handed gauge bosons. This rate is much larger than our estimate of $\Gamma_{0}(\chi \rightarrow l l \overline{\phi \phi})$ if $g_{R} \sim g$, and the two-photons decay mode gives the dominant dilution factor of the lepton asymmetry. 
This constraint arises only because the scalar is related to the same symmetry breaking scale as the corresponding massive gauge bosons. In principle, it should be possible to construct a model with a light neutral scalar that can only decay into light sterile right-handed fermions and heavy Majorana neutrinos, for instance by adding more scalar representations, but as this detracts from the simple gauge structure of the model, we will not pursue this here.

\section{Conclusions}

In a search for a clarification of the leptogenesis scheme, we have analysed a situation in which the Majorana neutrinos appear only as intermediate states. We have computed the lepton asymmetry without need to worry about defining propagation eigenstates for the unstable Majorana particles while including the $\mathrm{CP}$ violating effect from both the vertex and self-energy one-loop corrections. We have shown in section 2 that this very simple, but accordingly ad hoc scheme provides not only a consistent but also efficient mechanism of leptogenesis. The embedding in a realistic minimal gauged extension of the SM is however problematic. In particular, the addition of gauge degrees of freedom gives more dilution of the lepton asymmetry than is admissible. We have not considered more elaborate phenomenological models because our main interest was to address a question of principle -how to include all the $\mathrm{CP}$ violationg effects in a self-consistent an systematic framework. Obviously, a more general framework, that would allow to compute $\mathrm{CP}$ violation effects with Majorana neutrinos starting from first principles, and including the influence of a medium (like in the Early Universe) would be most welcome. 


\section{Acknowledgments}

This work was partially supported by the IISN (Belgium), and by the Com-

munauté Française de Belgique - Direction de la Recherche Scientifique programme ARC.

\section{References}

[1] M. Fukugita and T. Yanagida, Phys. Lett. 174B, 45 (1986).

[2] A. Cohen, D. Kaplan and A. Nelson, Ann. Rev. Nucl. Part. Sci. 43, 27 (1993).

[3] A.D. Sakharov, JETP Lett. 5, 24 (1967)

[4] see for instance R. Kolb and M. Turner, The Early Universe, (AddisonWesley, Redwood City, CA, 1990), and references therein.

[5] A. Yu. Ignatiev, V.A. Kuzmin and M.R. Shaposhnikov, JETP Lett. 30, 688 (1979).

[6] V. Weisskopf and R.P. Wigner, Z. Phys. 63, 54 (1930).

[7] F.J. Botella and J. Roldan, Phys.Rev. D44, 966 (1991).

[8] J. Liu and G. Segrè, Phys.Rev. D48, 4609 (1993); J. Liu and G. Segrè, ibid D49, 1342 (1994); Jiang Liu, Gino Segre, Phys. Lett. B338, 259 (1994).

[9] M. Flanz, E. A. Paschos and U. Sarkar, Phys. Lett. B345, 248 (1995);

M. Flanz, E. A. Paschos, U. Sarkar and J. Weiss, ibid B389, 693 (1996).

[10] L. Covi and E. Roulet, Phys.Lett. B399, 113 (1997). 
[11] A. Pilaftsis, Phys. Rev. D56, 5431 (1997).

[12] W. Buchmüller and M. Plümacher, Phys. Lett. B431, 354 (1998).

[13] E. Roulet, L. Covi and F. Vissani, Phys. Lett. B424, 101 (1998).

[14] M. Flanz, E. A. Paschos, Phys. Rev. D58, 113009 (1998).

[15] G.C. Branco, M.N. Rebelo, J.I. Silva-Marcos, hep-ph/9810328

[16] J. Ellis, M. K. Gaillard and D.V. Nanopoulos, Nucl.Phys. B106, 292 (1976) 